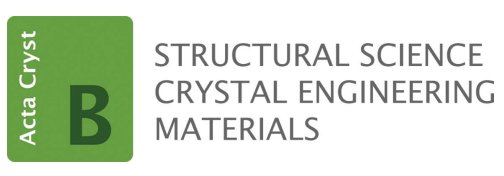

ISSN 2052-5206

Keywords: editorial; crystal growth; journal scope; crystal engineering; materials; structural science; high pressure; in situ studies.

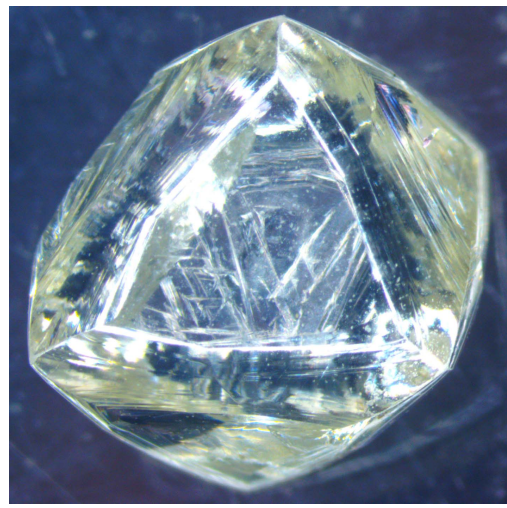

(C) 2021 International Union of Crystallography

\section{A new home for studies of crystal growth and characterisation}

\author{
Alexander J. Blake, ${ }^{\mathrm{a} *}$ Marc de Boissieu, ${ }^{\mathrm{b}}$ Ashwini Nangia, ${ }^{\mathrm{c}}$ Elena Boldyreva, ${ }^{\text {d,e }}$ \\ Andrzej Katrusiak, ${ }^{f}$ Tatyana B. Bekker ${ }^{\mathrm{g}}$ and Karah E. Knope ${ }^{\mathrm{h}}$

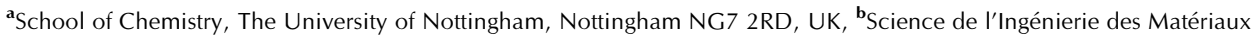 \\ et Procédés (SIMaP), 38402 Saint Martin d'Hères CEDEX, France, 'School of Chemistry, University of Hyderabad, \\ Hyderabad 500 046, India, 'Boreskov Institute of Catalysis, Russian Academy of Sciences, Novosibirsk 630090, Russian \\ Federation, ' Novosibirsk State University, Novosibirsk 630090, Russian Federation, '́ Department of Materials Chemistry, \\ Adam Mickiewicz University, 61-614 Poznan, Poland, Institute of Geology and Mineralogy, Siberian Branch of Russian \\ Academy of Science, Novosibirsk, Russian Federation, and hepartment of Chemistry, Georgetown University, \\ Washington, DC 20057, USA. *Correspondence e-mail: alexanderjohnblake@outlook.com
}

Crystal growth is fundamental to much of crystallography, but in contrast to articles describing the crystallization of biological macromolecules, which are catered for by Acta Crystallographica Sections $D$ and $F$, those dealing with the growth of crystals of nonbiological 'small' molecules or those of extended organic, inorganic or hybrid materials have lacked equally obvious routes to publication in IUCr journals. It is therefore timely to emphasize that Acta Crystallographica Section B offers such a route, by devoting a segment of the journal to relevant submissions. The ambition is to attract high-quality articles on crystal growth that are of broad interest and high impact, and there will be no arbitrary limit on the number of such articles.

Studies of crystal growth submitted to Acta Crystallographica Section B should align with the general scope of the journal, the subtitle of which is 'Structural Science, Crystal Engineering and Materials'. Structural science studies typically begin with the preparation of a crystal or other sample suitable for a diffraction experiment, but the importance of crystal growth in this context is far greater than simply producing a well diffracting crystal. Significant efforts are devoted to studying the mechanisms of crystal growth, as well as the melting, sublimation and dissolution behaviour of crystal forms. Moreover, control of crystal growth in aspects of both morphology (shape and size) and crystal polymorphism, based on the interplay of thermodynamics and kinetics, is fundamental to understanding the factors that determine crystal structure at the atomic, molecular, and macroscopic levels, and their impact on physical and chemical properties. Crystal engineering deals with the design, synthesis and growth of solid-state molecular assemblies with the aim of producing materials with defined desirable properties, since the bulk properties of many molecular materials are determined by the solid-state arrangement of the molecules. The method is based on a comprehensive understanding of intermolecular interactions and how they can be utilized to assemble extended crystal structures. A very wide range of intermolecular interactions, including hydrogen bonding, halogen bonding, $\pi-\pi$ interactions and van der Waals interactions have been exploited for crystal growth in crystal engineering. The range of materials and properties (e.g. hydrogen storage, catalysis, gas separation, pharmaceuticals, optoelectronic materials) which have been designed is so extensive that even a much longer list would still not be representative. Materials are often composed of crystals, either as individual large single crystals with sizes in the range of centimetres and even metres, or with polygrain structures. Even at the other extreme of the size range, nanocrystals also need to be grown prior to being studied. Growing single or polygrain crystals, mastering their defect concentration (either to avoid defects or to engineer defects for given physical properties) is at the heart of material science. The understanding of the growth process, its modelling, the detailed characterization of the atomic structure of the obtained crystals with defects, stoichiometry etc., are key prerequisites to achieving an understanding of structure-property relationships.

Crystallization applies to most of condensed matter, including the naturally-occurring minerals, metals in almost infinitely various forms, organic compounds and many others. 
Recently we have witnessed enormous progress in crystallization methods, such as the special environments capable of reproducing in the laboratory the thermodynamic conditions of interstellar space, through hydrothermal baths, to the centre of Earth, extremely slow annealing in vacuum and ultrafast nucleation under terapascal (TPa) shocks, the size effects remarkably evident in nanotechnology, refined co-crystallizations, as well as the advanced methods of investigating the most subtle structural effects by super-intense microbeams combined with highly sensitive detectors. All this on one hand provides new information for better understanding the science and art of crystallization, and on the other hand opens new routes for obtaining new unprecedented forms of matter that can foster progress in scientific and technological development.

Some studies of crystal growth from more theoretical viewpoints have appeared in Acta Crystallographica Section A or Journal of Applied Crystallography, and we expect this to continue. However, as there are likely to be borderline cases, potential authors are encouraged to consult the Notes for Authors or contact a Main or Section Editor to discuss the most relevant journal for their submission.

The products of the crystal growth procedures, which may be single crystals, microcrystals, powders, nanocrystals, defect structures, incommensurate structures, etc., must be characterized by direct structural analysis based on diffraction or atomic scale imaging techniques. An article which describes a technique for growing crystals of a material but lacks a distinct crystallographic component or a complete and convincing structural characterization is unlikely to be suitable for Acta Crystallographica Section B. Other existing journal criteria also apply to crystal growth papers: these include relevance to the scope of the journal, clear and complete descriptions of experimental procedures, evidence that demonstrates that the method described is reproducible and whether the resulting sample is homogeneous or otherwise.

To emphasize that Acta Crystallographica Section $B$ actively welcomes such submissions, the Notes for Authors contains a completely new section (\$3) on crystal growth papers which present some examples of possible research topics. Our interest in publishing such papers is further signalled by the recent appointment of two Co-editors, Tatyana Bekker (Institute of Geology and Mineralogy, Siberian Branch of Russian Academy of Science, Novosibirsk, Russian Federation) and Karah Knope (Department of Chemistry, Georgetown University, Washington, DC, USA), who are experts in crystal growth. We are commissioning feature articles which will explain further the types of papers the journal is seeking to attract. Examples of crystallization under unusual conditions (high-pressure, cryogenic, levitation, confined medium, template-assisted, electric and magnetic field, laser irradiation, etc.) are welcome. A special collection of recent articles on crystal growth will be collated, followed later by a special issue comprising new papers.

These initiatives and developments demonstrate that Acta Crystallographica Section $B$ is now a natural home for publications related to studies of crystal growth, and we invite the relevant communities to submit their work to the journal. 